\title{
A gigantic desmoplastic fibroma resembling a malignant neoplasm: an intriguing case report
}

\begin{abstract}
Desmoplastic fibromas of bone have been described as a rare, non-metastasizing, locally aggressive, benign lesions that histologically resemble a desmoid tumour of the soft tissue. Since his original description, a number of case reports have been published, as many as 150 . They are very common in mandible but here we report a fascinating case in a 21 year old young boy in the body of mandible which has grown to gigantic proportion in a short period of time and showed very aggressive radiographic presentation unlike conventional cases. In the present case, we have described clinical, radiological and histopathological features of Desmoplastic fibroma in detail. The gigantic size and aggressiveness of the lesion are the highlights in this case report.
\end{abstract}

Keywords: spindle cell neoplasm, desmoplastic fibroma, fibrosarcoma, hot spot mutations

\author{
Volume 4 Issue 3 - 2017

\section{Prashant Ramachandra, Vandrangi Sameer Kumar, Narendra Kumar Yandrapu, Sravya Reddy} \\ Department of oral and maxillofacial pathology, India
}

Correspondence: Prashant Ramachandra, Department of oral and maxillofacial pathology, Krishnadevaraya college of denta sciences, Bangalore, India, Email prashantopath@gmail.com

Received: May 29, 2017 | Published: October 27, 2017
Abbreviations: OPG, orthopantomogram; CT, computed tomography; DF, desmoplastic fibroma; WHO, world health organization

\section{Introduction}

Desmoplastic fibromas of bone has been described as a rare, nonmetastasizing, locally aggressive, benign lesions that histologically resembles a desmoid tumour of the soft tissue. ${ }^{1,2}$ It was initially described by Jaffe in 1958 who highlighted the histological resemblance to aggressive fibromatosis (desmoid tumour). ${ }^{3}$ Since his original description, approximately 150 case reports have been published. ${ }^{1}$ Desmoplastic fibromas are very common in mandible as they constitute approximately $86 \%$ of total cases cases ${ }^{4}$ but the current case report illustrates an interesting neoplasm which has grown to gigantic proportion in a short period of time and showed very aggressive radiographic presentation unlike conventional cases.

\section{Case report}

A 21year old male patient presented with a history of swelling in left jaw region since 6months which actually manifested after extraction of two of his posterior teeth. The swelling is asymptomatic but patient is worried because of abnormal growth in and around the extraction site. On extraoral examination there was a firm swelling present on the left side of the jaw which is diffuse, fixed to the body of mandible. Skin over the swelling was normal (Figure 1).

Intra-orally a huge swelling is present in the labial vestibule extending from mesial aspect of 33 to 38 and also extending towards the floor of the mouth. It is appearing fleshy with overlying mucosa exhibiting pale and erythematous areas and vestibular obliteration is evident. It is solitary, multi lobulated, well circumscribed swelling, irregular in shape, measuring about $6 \times 5 \times 4 \mathrm{~cm}$ in size (Figure 2). On palpation swelling was very firm, nontender, and rough. Bone seems evidently involved on palpating lower border. Considering the huge size of the mass and rapid growth the clinical differentials we considered were odontogenic tumors such as Ameloblastic fibroma, Odontogenic Myxoma, Ameloblastoma; Primary intraosseous Oral Squamous cell carcinoma, Intraosseous Muco-epidermoid Carcinoma, and other Sarcomas such as Osteosarcoma, Ewing's Sarcoma or Fibrosarcoma.

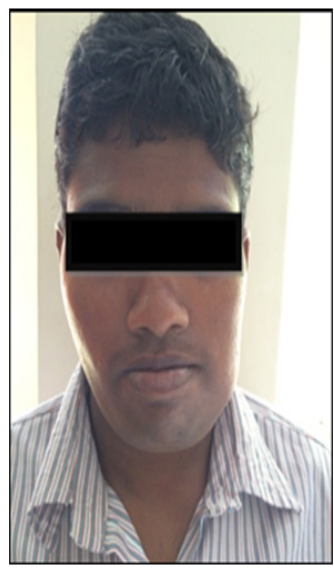

Figure I Frontal photograph showing diffuse swelling over left lower jaw.

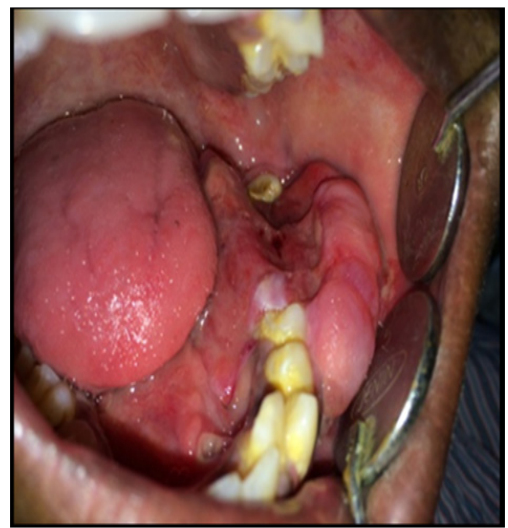

Figure 2 Intra oral photograph showing multi-lobular overgrowth in buccal vestibule extending towards floor of mouth.

Orthopantomogram (OPG) showed a well defined radiolucent lesion extending from 35 to 38 involving lower border of mandible. 
The lesion has displaced the roots of 35 and 38 . There is no evident root resorption. Scalloped areas were prominent in the lower border of mandible. There were no calcified foci in the radiolucent area. There is trace of mandibular canal posterior to 38 but it appears to be involved or pushed in the lesional area (Figure 3).

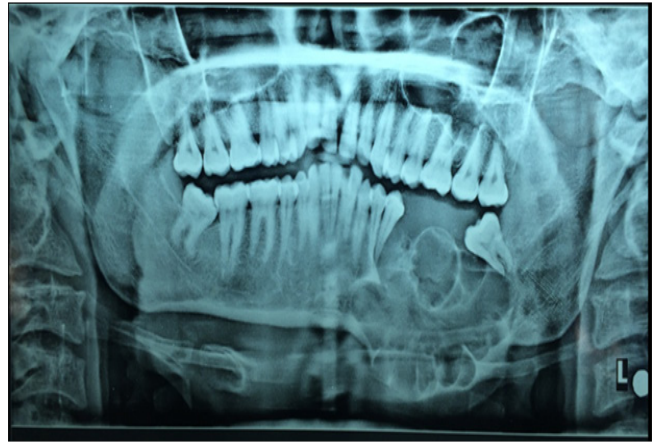

Figure 3 Orthopantamograph showing well defined radiolucent lesion involving lower border of mandible and prominent scalloped areas.

Coronal CT scan of the lesion showed irregular radiodense areas in left mandible which were extending into soft tissues and floor of mouth (Figure 4). 3-D CT Scan shows multiple perforations of irregular sizes and shapes (Figure 5). Axial- CT shows expanded bony cortices in the form of locules which are characteristic of benign lesion (Figure 6) but overall findings indicate a frank malignant lesion. Thus we have considered intraosseous Squamous cell carcinoma, Odontogenic Carcinomas and sarcomas, Intraosseous Mucoepidermoid carcinoma, Other Sarcomas such as Ewings Sarcoma, Osteosarcoma as radiological differentials. Left segmental mandibulectomy was done under general anaesthesia (Figure 7).

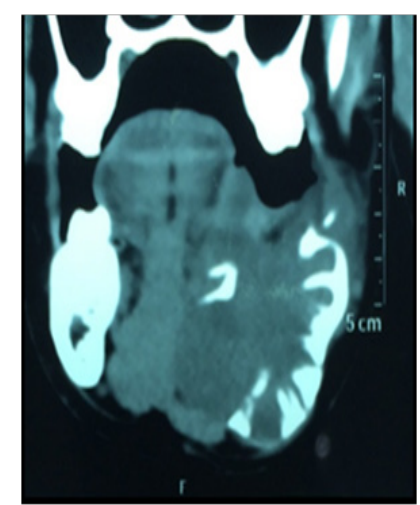

Figure 4 Coronal CT scan showing radiodense areas extending into soft tissues and floor of mouth.

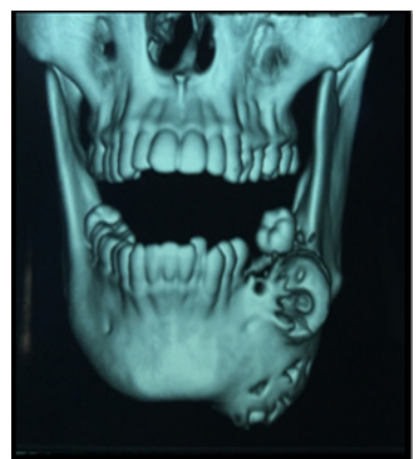

Figure 5 3-Dimensional CT scan depicting multiple perforations on buccal and inferior surface of body of mandible.

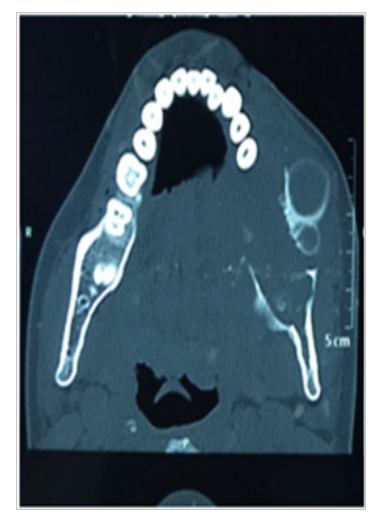

Figure 6 Axial- CT shows expanded bony cortices in the form of locules.

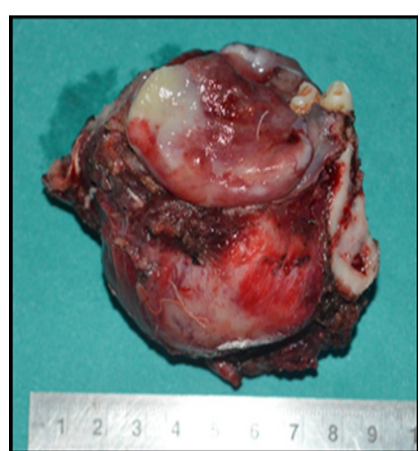

Figure 7 segmental mandibulectomy specimen measuring $9 \times 6 \times 5 \mathrm{~cm}$.

Microscopic examination revealed presence of well encapsulated lesional tissue mass composed of fascicles of uniform cells (Figure 8). On closer examination, the neoplasm is composed of spindle cells in short fascicles and whorls (Figure 9). The fusiform cells depict indistinct cell borders, scanty cytoplasm and slender tapering nuclei without any atypia (Figure 10). Few normal mitoses are present. Pockets of chronic lympho-plasmacytic infiltrate and scattered mast cells are seen (Figure 11).

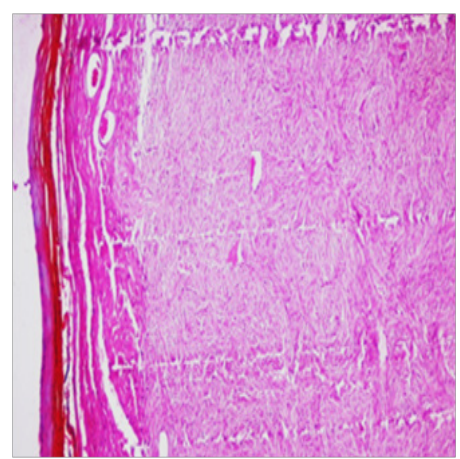

Figure 8 Low power histopathologic image shows an encapsulated lesion.

\section{As of now it is quite clear that}

It is primarily bone pathology (Intra-osseous Origin): because a pathologic process beginning within bone and secondarily extending into soft tissues typically follows inside-out principle, where the soft tissue mass is smaller than the degree of bone destruction.

It's a Spindle cell neoplasm: Histopathologic differentials which we have considered based on histological findings are Cellular benign fibrous histocytoma, fibromatosis, nodular fascitis, low grade fibrosarcoma, inflammatory myofibroblastic tumor. Immunohistochemis- 
try was performed and the neoplastic cells are negative for desmin, S-100, CD-34, EMA and Ki-67 is very low i.e less than 1/10 HPF. So based on the morphological features and exclusion of sarcomas based on immunohistochemistry findings, the final diagnosis of Desmoplastic fibroma was given.

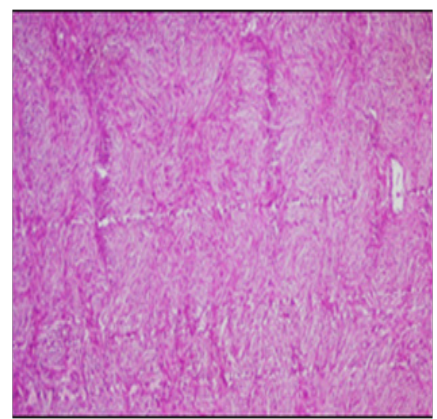

Figure 9 Low power image shows spindle cells in short fascicles and whorls.

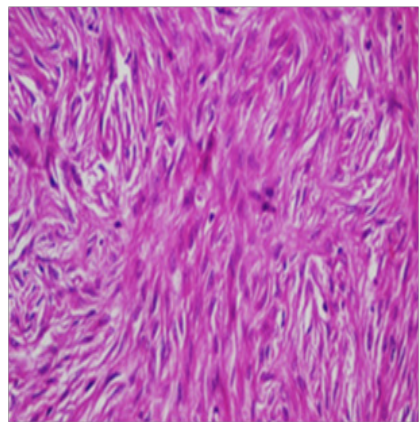

Figure 10 High power image shows fusiform cells depicting indistinct cel borders, scanty cytoplasm and slender tapering nuclei without atypia.

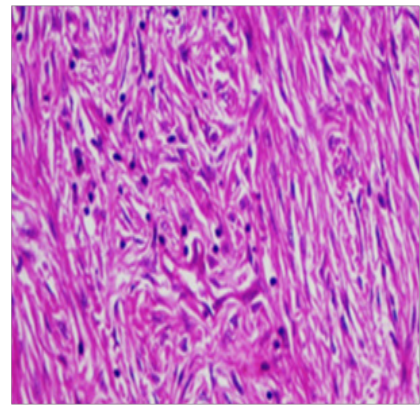

Figure II High power image shows pockets of chronic lympho-plasmacytic infiltrate and few scattered mast cells.

\section{Discussion}

The desmoplastic fibroma (DF) is a rare, fibroblastic lesion of bone that resembles histological counterpart of desmoid tumor of soft tissue. Although classified as benign, it frequently demonstrates aggressive behavior, often causing tooth mobility, extensive bone destruction, as seen in the current case report and has a moderate to high recurrence rate.${ }^{6,7}$ Activating CTNNB1 hotspot mutations or APC mutations are driving events ${ }^{8,9}$ for these locally aggressive tumors.

According to World Health Organization (WHO) Desmoplastic fibroma is defined as a locally aggressive (myo) fibroblastic lesion of bone. It is also known as the desmoid tumor of bone. It is seen in patients aged less than 30 years. Desmoplastic fibromas are very common in mandible as they constitute approximately $86 \%$ cases in mandible ${ }^{4}$ with a predilection for the ramus and angle region, Usually the lesions are slow growing ${ }^{6}$ and radiographically well defined but the presence of such a massive lesion which has grown in such a short time span with multiple buccal and lingual cortical plate perforations and infiltration of floor of mouth and soft tissues is a very rare finding ${ }^{10}$ when we compare current case report with past cases.

The fact that this did not happen to be a malignancy was such a relief for the patient. So not every aggressive appearing neoplasm should be a matter of worry for the clinician or patient. Current case report reiterates that histologic examination is the gold standard for diagnosis and immunohistochemistry adds so much value to the diagnosis not only when it is positive but also when there is no staining. The patient is under follow up and there was no recurrence as of now.

\section{Conclusion}

It is important to include desmoplastic fibroma in the list of differential diagnoses of extremely aggressive neoplasms in the jaw because failure to do so can lead to significant patient morbidities.

\section{Acknowledgements}

None.

\section{Conflict of interest}

Author declares that there is no conflict of interest.

\section{References}

1. Shukul VK, Saxena S, Shankar BG. Desmoplastic fibroma: mandible. Medical Journal Armed Forces India. 2004;60(3):307-309.

2. Shi H, Wang P, Wang S, et al. Desmoplastic fibroma of the mandible. Dentomaxillofacial Radiology. 2014:37(7)

3. Jaffe HL. Tumors and tumorous conditions of the bones and joints. Academic Medicine. 1959;34(1):72.

4. El Naggar Ak, Chan JKC, Grandis JR, et al. Classification of Tumours. 4th ed. World Health Organization. Lyon: IARC press; 2017.

5. Evans S, Ramasamy A, Jeys L, et al. Desmoplastic fibroma of bone: A rare bone tumour. J Bone Oncol. 2014;3(3):77-79.

6. Woods TR, Cohen DM, Islam MN, et al. Desmoplastic fibroma of the mandible: a series of three cases and review of literature. Head Neck Pathol. 2015;9(2):196-204.

7. De Vito MA, Tom LW, Boran TV, et al. Desmoplastic fibroma of the mandible. Ear, nose, \& throat Journal. 1989;68(7):553-556.

8. Horvai AE, Jordan RC. Fibro-Osseous Lesions of the Craniofacial Bones: $\beta$-Catenin Immunohistochemical Analysis and CTNNB1 and APC Mutation Analysis. Head Neck pathol. 2014;8(3):291-297.

9. Flucke U, Tops BB, Diest PJ, et al. Desmoid-type fibromatosis of the head and neck region in the paediatric population: a clinicopathological and genetic study of seven cases. Histopathology. 2014;64(6):769-776.

10. Inwards CY, Krishnan Unni K, Beabout JW, et al. Desmoplastic fibroma of bone. Cancer. 1991;68(9):1978-1983. 